\title{
Erratum
}

Z Gerontol Geriat 2021 · 54:305

https://doi.org/10.1007/s00391-021-01889-5

Published online: 21 April 2021

(c) The Author(s) 2021

Julia Schneider' $\cdot$ Mara Gkioka ${ }^{1,2} \cdot$ Sotirios Papagiannopoulos ${ }^{3}$. Despina Moraitou ${ }^{4} \cdot$ Brigitte Metz $^{5} \cdot$ Magdalini Tsolaki $^{6} \cdot$ Andreas Kruse $^{7}$. Birgit Teichmann'

${ }^{1}$ Network Aging Research, Heidelberg University, Heidelberg, Germany

${ }^{2}$ School of Medicine, Aristotle University of Thessaloniki, University Campus, Thessaloniki, Greece ${ }^{3}$ 3rd Department of Neurology, Aristotle University of Thessaloniki, Thessaloniki, Greece

${ }^{4}$ Lab of Psychology, Section of Experimental \& Cognitive Psychology, School of Psychology, Aristotle University of Thessaloniki, Thessaloniki, Greece

${ }^{5}$ Klinik für Geriatrie und Geriatrisches Zentrum Karlsruhe, Diakonissenkrankenhaus Karlsruhe-Rüppurr, ViDia Christliche Kliniken Karlsruhe, Karlsruhe, Germany

${ }^{6} 1$ st Department of Neurology, AHEPA University Hospital, Thessaloniki, Greece

${ }^{7}$ Insitute of Gerontology, Heidelberg University, Heidelberg, Germany

\section{Erratum to: Expectations of nursing personnel and physicians on dementia training}

\section{A descriptive survey in general hospitals in Germany and Greece}

\section{Supplementary Information \\ The online version of this article (https://doi. org/10.1007/s00391-021-01889-5) contains supplementary material, which is available to authorized users.}

\section{Erratum to:}

Z Gerontol Geriat 2019

https://doi.org/10.1007/s00391-019-

01625-0

Please note the correct tables of the electronic supplementary material. The original article has been corrected.

\section{Corresponding address}

\section{Julia Schneider}

Network Aging Research, Heidelberg University Bergheimer Straße 20, 69115 Heidelberg, Germany

schneider@nar.uni-heidelberg.de

\section{Mara Gkioka}

School of Medicine, Aristotle University of Thessaloniki, University Campus

54124 Thessaloniki, Greece gkioka@nar.uni-heidelberg.de

Open Access. This article is distributed under the terms of the Creative Commons Attribution 4.0 International License (http://creativecommons.org/licenses/by/ 4.0/), which permits unrestricted use, distribution and reproduction in any medium, provided you give appropriate credit to the original author(s) and the source, provide a link to the Creative Commons license, and indicate if changes were made. 